\title{
Circular dichroism in non-chiral metal halide perovskites
}

\author{
Peter C. Sercel, ${ }^{1,2, *}$ Zeev Valy Vardeny, ${ }^{3}$ and Alexander L. Efros ${ }^{4}$ \\ ${ }^{1}$ Department of Applied Physics and Materials Science, \\ California Institute of Technology, Pasadena, California 91125, USA \\ ${ }^{2}$ Center for Hybrid Organic Inorganic Semiconductors for Energy, \\ 15013 Denver West Parkway, Golden, CO 80401, USA \\ ${ }^{3}$ Department of Physics and Astronomy, \\ University of Utah, Salt Lake City, UT 84112, USA \\ ${ }^{4}$ Center for Computational Materials Science, \\ U. S. Naval Research Laboratory, Washington DC 20375, USA
}

We demonstrate theoretically that non-chiral perovskite layers can exhibit circular dichroism (CD) in the absence of a magnetic field and without chiral activation by chiral molecules. The effect is shown to be due to splitting of helical excitonic states which can form in structures of orthorhombic or lower symmetry that exhibit Rashba spin effects. The selective coupling of these helical exciton states to helical light is shown to give rise to circular dichroism. Polarization dependent absorption is shown to occur due to the combined effect of Rashba splitting, in-plane symmetry breaking, and the effect of the exciton momentum on its fine structure, which takes the form of Zeeman splitting in an effective magnetic field. We calculate significant CD with an anisotropy factor of up to $30 \%$ in orthorhombic perovskite layers under off-normal top illumination conditions, raising the possibility of its observation in non-chiral perovskite structures.

Keywords: chirality, circular dichroism, lead halide perovskite, hybrid organic inorganic perovskite, Rashba effect, exciton fine structure, nanoplatelet

\footnotetext{
* psercel@caltech.edu
} 
An object, a molecule or a crystal structure is chiral if it is distinguishable from its mirror image; that is, it cannot be superimposed onto the original by any sequence of pure rotations or translations. $\stackrel{\underline{1}}{\text { The }}$ Thiversally recognized example of chiral objects are human hands because the left hand is a non-superimposable mirror image of the right hand, no matter how the two hands are oriented. In nature, chiral molecules and crystal structures exist in two unmixed geometrical forms (two enantiomers) that are mirror images of each other because their low symmetry does not allowed gradual transformation from one to the other.

The growing attention to chiral materials is connected with their potential applications in the area of chiral optoelectronics and spintronics. Chirality is associated with the phenomenon of circular dichroism $(\mathrm{CD})^{2}$ since enantiomer pairs absorb left and right circularly polarized light differently $\underline{\underline{3}} \underline{\underline{\underline{T}}}$, and enantiomers can also emit circularly polarized light in the absence of an external magnetic field. $\underline{\underline{8}} \underline{\underline{10}}$ Recently chiral materials have been used in spintronic devices as a spin filters due to chiral-induced spin selectivity $\underline{\underline{11}} \underline{\underline{13}}$

Chiral non-centrosymmetric molecules and crystals can be categorized into 65 Sohncke space groups within different Bravais lattices and point groups (see for example the review by Long et al. in Ref. 14). Chirality is very common among natural organic compounds, such as amino acids, sugar molecules, peptides and DNA. However, inorganic materials that show CD or circular birefringence are relatively rare. The most famous among them, and the first discovered, is quartz: $\stackrel{15}{\underline{1}}$ Other examples include zinc blende semiconductors under uniaxial stress. $\underline{\underline{16}}$

Semiconductor inorganic nanomaterials, such as quantum dots (QDs) and nanoplatelets have been shown however to exhibit CD after "chiral activation" of their surface by chiral organic molecules $\underline{\underline{4}, 17-\underline{19}}$ An excellent recent review $\underline{\underline{20}}$ discusses different induction mechanisms that have been suggested for achieving this: (i) chiral-organic-molecule-induced crystallization of nanostructures into a chiral structure $\stackrel{21}{\underline{1}}$ (ii) surface-chiral-organic-molecule- induced chiral distortion of the surfaces of QDs $\stackrel{22}{\underline{2}}$ and (iii) electronic interactions between chiral organic molecules and QDs. $\frac{18}{\underline{1}}$ Chiral activation of hybrid organic - inorganic perovskites (HOIPs) by incorporation of chiral organic molecules within the inorganic framework of these perovskites is the most successful realization of this idea. $\underline{\underline{23}}$ The first demonstration

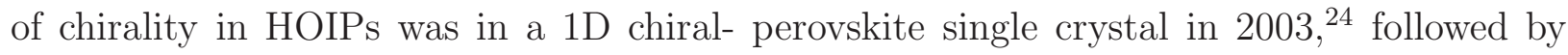
2D chiral- perovskite single crystals in 2006 $\underline{\underline{25}}$ Homogeneous chiral films can be fabricated 
from perovskites by using various film-coating methods underscoring the practically of this approach. $\underline{\underline{20}}$

Recently, CD has been observed in QDs and nanorods which have not been activated by chiral molecules $\underline{\underline{26}} \underline{\underline{28}}$ This is surprising since observation of chiro-optic activity in randomly oriented nanoparticles requires chirality. To explain this observation the authors of Ref. 26 suggested that the QDs have chiral defects such as screw dislocations, which affect the optical properties of small size CdSe/ZnS quantum dots, inducing the CD. In contrast, in Ref. 29, elliptical polarization in the photoluminescence of single $\mathrm{CsPBB}_{3}$ nanocrystals at zero magnetic field was attributed to inversion symmetry breaking and the Rashba effect.

It has long been known that symmetry considerations permit optical activity and CD in certain non-chiral crystal structures, namely, the four non-chiral crystal classes corresponding to point groups $C_{s}, C_{2 v}, S_{4}$, and $D_{2 d}(m, m m 2, \overline{4}$, and $\overline{4} 2 m$ in Hermann- Mauguin notation, respectively) $\stackrel{30}{\underline{3} \underline{31}}$ The first observation of this phenomenon was made only in 1967 in $\mathrm{AgGaS}_{2} \underline{\underline{31}}$ because the optical activity of these non-chiral crystal structures is weak. To understand why chiro-optic effects might be observed in these non-chiral structures, let us consider a crystal with point symmetry $C_{2 v}$. Although the crystal is non-chiral because it has two mirror planes, if we consider the effect of the measuring light as shown in Fig. 1, we recognize that the symmetry of the complete light-matter system, without orientational averaging, is in fact chiral due to the symmetry breaking associated with the directionality of the light. Such light-induced chirality, which has been termed "extrinsic chirality" $\underline{\underline{32}}$, was recently demonstrated to cause chiro-optic effects in planar metamaterials, $\underline{32}, 33$ although the planar metamaterial systems exhibit reversal of handedness when illuminated from opposite directions $\underline{\underline{33}, \underline{34}}$

In this paper we suggest a specific physical mechanism that leads to large CD in non-chiral metal halide perovskite crystals with broken inversion symmetry, in the absence of any chiral molecules at their surface. Since many perovskites adopt an orthorhombic crystal structure at low temperature, we consider an orthorhombic system with inversion symmetry breaking normal to the surface along a two-fold rotation axis: The structure possesses non-chiral $C_{2 v}$ point symmetry (see Figure 1) and is expected to exhibit spin splitting due to the large spin orbit coupling in these systems. Calculations of excitonic light absorption conducted in quasi-two dimensional metal halide perovskites using experimentally determined Rashba spin-orbital splitting parameters $\frac{35}{\underline{36}}$ show CD with an anisotropy factor $\underline{\underline{14}}$ of up to $30 \%$. 
The effect is shown to be due to splitting of helical excitonic states, which can exist only in structures of orthorhombic (or lower) symmetry with inversion symmetry breaking. The selective coupling of these helical states to helical light gives rise to CD.

A

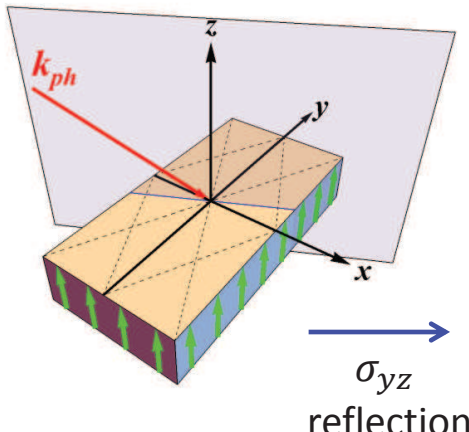

B

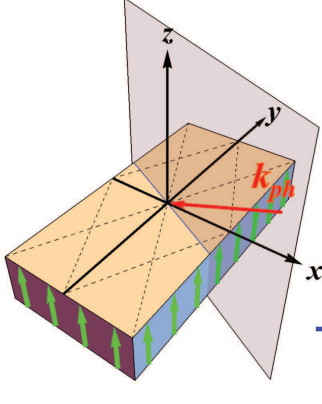

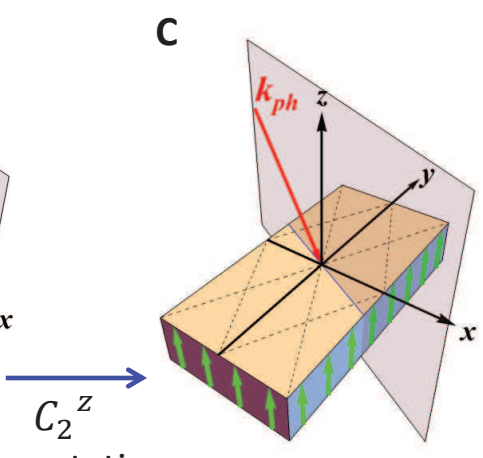

rotation

Figure 1. Schematic showing how a non-chiral structure can exhibit chiral behavior when interacting with light. The figure depicts light (shown via its wave vector $\boldsymbol{k}_{p h}$ ) incident on a non-chiral perovskite structure with orthorhombic point symmetry $C_{2 v}$ (depicted as a slab with a two-fold rotational symmetry about the $\hat{z}$ axis). The structure is assumed to have inversion asymmetry in the vertical $\hat{z}$ direction, depicted via the vertical green arrows in the slab, while the two vertical mirror planes axes are $\sigma_{x, z}$ and $\sigma_{y z}$. In the absence of light, the system is non-chiral because of its mirror symmetries. On the other hand, the system structure + light, shown in panel A, with light in a plane of incidence rotated at an arbitrary angle from either of the vertical mirror planes, is chiral: Its mirror image, panel B, cannot be restored to the original by any sequence of rotations or translations. As an example, panel $\mathrm{C}$ shows the effect of two fold rotation about the $\mathrm{z}$ axis. 


\section{EXCITONS IN PEROVSKITE LAYERS}

Let us consider the problem of an exciton in a semiconductor with parabolic conduction and valence bands. Introducing the relative electron-hole coordinates $\boldsymbol{r}=\boldsymbol{r}_{e}-\boldsymbol{r}_{h}$ and the coordinates of the exciton center of mass, $\boldsymbol{R}=\left(m_{e} \boldsymbol{r}_{e}+m_{h} \boldsymbol{r}_{h}\right) / M$, where the exciton translational mass $M=m_{e}+m_{h}$ is equal to the sum of the electron, $m_{e}$, and hole, $m_{h}$ effective masses, respectively, we can describe the exciton energy as a sum of Hamiltonians that describe the relative electron-hole motion, $\hat{H}_{0, \mathrm{REL}}$, and the exciton center of mass (COM) motion $\hat{H}_{0, \mathrm{COM}}$ :

$$
\hat{H}_{0}=\hat{H}_{0, \mathrm{COM}}+\hat{H}_{0, \mathrm{REL}}=\frac{\hat{\boldsymbol{P}}^{2}}{2 M}+\left[\frac{\hat{\boldsymbol{p}}^{2}}{2 \mu}+V(|\boldsymbol{r}|)\right] .
$$

Here, $\hat{\boldsymbol{P}}=\hat{\boldsymbol{p}}_{e}+\hat{\boldsymbol{p}}_{h}$ is the COM momentum while $\hat{\boldsymbol{p}}=\left(m_{h} \hat{\boldsymbol{p}}_{e}-m_{e} \hat{\boldsymbol{p}}_{h}\right) / M$ is the relative coordinate momentum, and $\mu=\left(1 / m_{e}+1 / m_{h}\right)^{-1}$ is the reduced mass. As a result of this separation, the wave function of a free exciton can be written in the form of a Bloch wave. In the case of a quasi-2D perovskite, the wave function takes the form,

$$
\Psi_{\boldsymbol{K}, n, m ; j_{e}, j_{h}}\left(\boldsymbol{r}_{e}, \boldsymbol{r}_{h}\right)=u_{j_{e}}\left(\boldsymbol{r}_{e}\right) u_{j_{h}}\left(\boldsymbol{r}_{h}\right) \frac{1}{\sqrt{S}} e^{i \boldsymbol{K} \cdot \boldsymbol{R}} \phi_{n, m}\left(\boldsymbol{r}_{e}-\boldsymbol{r}_{h}\right) .
$$

In this expression, wave vector $\boldsymbol{K}$ is equal to $\boldsymbol{P} / \hbar, S$ is the surface area of the $2 \mathrm{D}$ layer and $u_{j_{e}}$ and $u_{j_{h}}$ are the band-edge periodic basis functions for the electron and hole, respectively. The indices $j_{e}, j_{h}$ in $u_{j_{e}}$ and $u_{j_{h}}$ represent in abbreviated fashion the angular momentum quantum numbers $j, j_{z}$ associated with the electron and hole Bloch functions. In Eq. (2) $\phi_{n, m}$ is the normalized wave function that describes the relative motion of the electron and hole and is characterized by principle and azimuthal quantum numbers $n$ and $m$. The energy spectrum of the 2D exciton consequently can be written as:

$$
E_{n,|m|}^{0}(\boldsymbol{K})=\mathcal{E}_{n,|m|}+\frac{\hbar^{2} \boldsymbol{K}^{2}}{2 M},
$$

where $\mathcal{E}_{n,|m|}$ are the eigenvalues associated with the internal exciton motion. In the absence of dielectric confinement, that is, when the dielectric constants of semiconductor, $\kappa$, and the surrounding medium are equal, the Coulomb potential can be written as $V(\rho)=e^{2} / \kappa \rho$, where $\rho=\left|\boldsymbol{r}_{e}-\boldsymbol{r}_{h}\right|$. In this limit, expressions for the energies and wavefunctions of $2 \mathrm{D}$ excitons are well known and can be found for example in Refs. 37,38. Due to the small value of the dielectric constant of organic materials, dielectric confinement can strongly 
enhance the electron-hole Coulomb interaction in hybrid organic-inorganic layered perovskite semiconductors. Analytical expressions for the Coulomb interaction potential $V(\rho)$ which account for dielectric confinement effects were derived by Ritova ${ }^{39}$ and Keldish $\stackrel{40}{\text {. The }}$ enhancement of the exciton binding energies for several low-energy transitions has been demonstrated in CdSe nanoplatelets $\underline{41}$

\section{RASHBA EFFECT IN A PEROVSKITE LAYER}

Let us now consider a metal halide perovskite with orthorhombic crystal symmetry and broken inversion symmetry in the $z$ direction perpendicular to the layer $\underline{36}$ Due to the strong spin-orbit coupling in metal halide perovskite semiconductors, the broken inversion symmetry generates Rashba terms that are linear in the electron and hole momenta. It was shown Ref. 42 that in general, for crystals that have orthorhombic symmetry with an arbitrary symmetry breaking direction a total of 12 terms (6 for electron operators, 6 for hole operators) are required for a general description of the Rashba effect for excitons. In the case that inversion symmetry is broken in the $z$ direction the Rashba Hamiltonian is significantly simplified and is described just by two Rashba coefficients, $\alpha_{x y}^{e}$ and $\alpha_{y x}^{e}$ for the conduction band and two Rashba coefficients, $\alpha_{x y}^{h}$ and $\alpha_{y x}^{h}$, for the valence bands:

$$
\hat{H}_{R}=\frac{\alpha_{x y}^{e}}{\hbar} J_{x} \hat{p}_{y}^{e}-\frac{\alpha_{y x}^{e}}{\hbar} J_{y} \hat{p}_{x}^{e}+\frac{\alpha_{x y}^{h}}{\hbar} s_{x} \hat{p}_{y}^{h}-\frac{\alpha_{y x}^{h}}{\hbar} s_{y} \hat{p}_{x}^{h}
$$

Here, $J_{x, y}$ and $s_{x, y}$ are the $x$ and $y$ Pauli operators corresponding to the angular momenta of the electron and hole, respectively, while $\hat{p}_{y, x}^{e, h}$ are the projections of the electron and hole momentum operators on the $x$ and $y$ axes. For analysis of the effect of Rashba terms on the free exciton, it is useful to express the electron and hole momentum operators in terms of COM and relative momenta. Using $\hat{\boldsymbol{p}}_{e}=\left(m_{e} / M\right) \boldsymbol{P}+\hat{\boldsymbol{p}}$ and $\hat{\boldsymbol{p}}_{h}=\left(m_{h} / M\right) \boldsymbol{P}-\hat{\boldsymbol{p}}$, we transform the Rashba Hamiltonian in Eq. (4) into a sum of Rashba Hamiltonians for the exciton center of mass motion, $\hat{H}_{R, \mathrm{COM}}$, and the electron -hole relative motion, $\hat{H}_{R, \mathrm{REL}}$, where

$$
\begin{aligned}
\hat{H}_{R, \mathrm{COM}}(\boldsymbol{P}) & =\frac{1}{M}\left[\left(m_{e} \frac{\alpha_{x y}^{e}}{\hbar} J_{x}+m_{h} \frac{\alpha_{x y}^{h}}{\hbar} s_{x}\right) P_{y}-\left(m_{e} \frac{\alpha_{y x}^{e}}{\hbar} J_{y}+m_{h} \frac{\alpha_{y x}^{h}}{\hbar} s_{y}\right) P_{x}\right] \\
\hat{H}_{R, \mathrm{REL}} & =\left(\frac{\alpha_{x y}^{e}}{\hbar} J_{x}-\frac{\alpha_{x y}^{h}}{\hbar} s_{x}\right) \hat{p}_{y}-\left(\frac{\alpha_{y x}^{e}}{\hbar} J_{y}-\frac{\alpha_{y x}^{h}}{\hbar} s_{y}\right) \hat{p}_{x}
\end{aligned}
$$


The effect of the Rashba Hamiltonian $\hat{H}_{R, \mathrm{REL}}$ on the fine structure of the exciton due to internal electron-hole motion can be described in the framework developed for the 3D exciton in Ref. 42. There, it was demonstrated that when Rashba terms exist in both the conduction and valence bands, an effective $\boldsymbol{J} \cdot \boldsymbol{s}$ interaction can invert the level order of the bright and dark exciton fine structure levels.

From now on however, for simplicity, we will consider Rashba terms only in the conduction band, neglecting the effect of spin orbit coupling in the valence band, assuming that $\alpha_{x y}^{h} \approx 0$ and $\alpha_{y x}^{h} \approx 0$. This is reasonable given that the valence band states transform with overall $s$

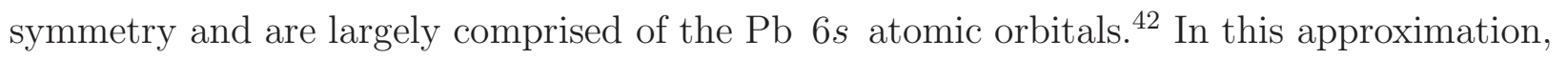
the Rashba terms $\hat{H}_{R, \mathrm{REL}}$ originating from the motion associated with the relative electronhole coordinate do not affect the exciton fine structure. $\stackrel{\underline{42}}{=}$ Only the Rashba terms associated with COM motion of the exciton enter into the present analysis. These terms, along with electron-hole exchange, determine the exciton fine structure. One can see from Eq.(5) that the Rashba Hamiltonian for the exciton center of mass motion, $\hat{H}_{R, \mathrm{COM}}$, does not mix states with different momenta, $\boldsymbol{P}=\hbar \boldsymbol{K}$, but it does mix the four angular momentum sublevels of the exciton fine structure created by the electron-hole exchange interaction. In this limit Rashba Hamiltonian $\hat{H}_{R, \mathrm{COM}}$ in Eq. (5) reduces to,

$$
\hat{H}_{R, \mathrm{COM}}(\boldsymbol{K})=\frac{m_{e}}{M}\left(\alpha_{x y} K_{y} J_{x}-\alpha_{y x} K_{x} J_{y}\right),
$$

where we have now dropped the superscript $e$ on the Rashba coefficients with the understanding that henceforth Rashba coefficients refer to the conduction band. Equation (6) can be rewritten as sum of 2D Rashba $\hat{H}_{R}^{2 D}(\boldsymbol{K})$ and 2D Dresselhaus $\hat{H}_{D}^{2 D}(\boldsymbol{K})$ terms that are familiar from studies of the effects of structural inversion asymmetry in $2 \mathrm{D}$ electron systems: $\underline{43}, \underline{44}$

$$
\hat{H}_{R, \mathrm{COM}}(\boldsymbol{K})=\hat{H}_{R}^{2 D}(\boldsymbol{K})+\hat{H}_{D}^{2 D}(\boldsymbol{K}),
$$

where

$$
\begin{array}{r}
\hat{H}_{R}^{2 D}(\boldsymbol{K})=\frac{m_{e} \alpha}{M}\left(K_{y} J_{x}-K_{x} J_{y}\right) \quad \text { (Pure 2D - Rashba); } \\
\hat{H}_{D}^{2 D}(\boldsymbol{K})=\frac{m_{e} \beta}{M}\left(J_{x} K_{y}+J_{y} K_{x}\right) \quad(\text { Pure } 2 \mathrm{D}-\text { Dresselhaus }),
\end{array}
$$

with $\alpha=\left(\alpha_{x y}+\alpha_{y x}\right) / 2$ and $\beta=\left(\alpha_{x y}-\alpha_{y x}\right) / 2$. In a system with cubic or tetragonal symmetry, $\alpha_{y x}=\alpha_{x y}=\alpha$ since these systems have four-fold rotational symmetry about 
the z-axis; the $x$ and $y$ directions are equivalent. We call this the "pure" Rashba limit. However, if the $x$ and $y$ directions are inequivalent, as is the case with orthorhombic $C_{2 v}$ point symmetry, then in general, the two Rashba coefficients may be distinct: $\alpha_{y x} \neq \alpha_{x y}$. In the extreme limit that $\alpha_{x y}=-\alpha_{y x}=\beta$ the Rashba part of Hamiltonian (6) vanishes, $\hat{H}_{R}^{2 D}(\boldsymbol{K}) \equiv 0$, and we have the pure 2D Dresselhaus Hamiltonian $\hat{H}_{D}^{2 D}(\boldsymbol{K})$.

To gain physical intuition on the splitting of the exciton levels, it is convenient to introduce an effective $\boldsymbol{K}$ dependent magnetic field $\boldsymbol{B}^{\text {eff }}(\boldsymbol{K})$ which allows us to write the Rashba Hamiltonian in Eq. [6] in the form of the Zeeman effect, $\hat{H}_{R, \mathrm{COM}}=g^{\text {eff }} \mu_{B} \boldsymbol{B}^{\text {eff }} \cdot \boldsymbol{J}$, where $\mu_{B}$ is the Bohr magneton, $g_{e f f}=m_{e} / M$, and the effective magnetic field is given by,

$$
\boldsymbol{B}_{e f f}(\boldsymbol{K}) \equiv \frac{1}{\mu_{B}}\left(\alpha_{x y} K_{y} \hat{x}-\alpha_{y x} K_{x} \hat{y}\right) .
$$

One can see that the orientation of this magnetic field depends strongly on $\boldsymbol{K}$. The magnitude of the effective magnetic field $B_{e f f}=\sqrt{\alpha_{x y}^{2} K_{y}^{2}+\alpha_{y x}^{2} K_{x}^{2}} / \mu_{B}$ may take on quite significant values. We estimate it using the conduction band Rashba energy of $40 \mathrm{meV}$ measured by Zhai et al. in the $2 \mathrm{D}$ layered perovskite $(\mathrm{PEA})_{2} \mathrm{PbI}_{4}(\mathrm{PEPI})$ and assume the electron and hole effective masses to be $0.25 m_{0} \stackrel{35}{\underline{3}}$ With light of free space wavelength $\lambda_{0}=500 \mathrm{~nm}$ incident at 45 degrees from vertical, assuming a refractive index $n=\sqrt{5}$, corresponding to a high frequency dielectric constant of $\epsilon_{\infty}=5$, which is typical of metal halide perovskites, the in-plane wave vector of excitons created by absorption is $K \sim 0.009 \mathrm{~nm}^{-1}$. This leads to an effective magnetic field of 24 Tesla. The resulting splitting of the exciton levels can be described using a basis of total angular momentum, $\boldsymbol{F}$, and its projection $F_{z_{B}}$, where the quantization axis $\boldsymbol{z}_{B}$ is the direction of the effective magnetic field: $\boldsymbol{z}_{B}=\boldsymbol{B}_{\text {eff }} / B_{\text {eff }}$.

Figure 2 shows vector field maps depicting the direction and relative magnitude of the effective magnetic field for the pure Rashba $(\alpha \neq 0, \beta=0)$ and the pure 2D Dresselhaus $(\alpha=0, \beta \neq 0)$ cases, as well as some mixed cases. From Eq. 10 and these field maps, we can recognize that in the pure Rashba case, the effective magnetic field is always perpendicular to the direction of exciton momentum $\boldsymbol{K}$. In the pure 2D Dresselhaus case by contrast, the effective magnetic field generally has a component along $\boldsymbol{K}$; on the lines $K_{x}= \pm K_{y}, \boldsymbol{B}_{\text {eff }}$ is co-linear with the direction of the vector $\boldsymbol{K}$. As a result, in this case, triplet excitons with angular momentum projection $F_{z_{B}}= \pm 1$ have helicity, a property which is defined as $\chi=\boldsymbol{F} \cdot \hat{\boldsymbol{n}}_{\boldsymbol{K}}$ where $\hat{\boldsymbol{n}}_{\boldsymbol{K}}=\boldsymbol{K} / K$. Excitons with angular momentum parallel $\left(F_{z_{B}}=+1\right)$ or antiparallel $\left(F_{z_{B}}=-1\right)$ to the direction of the momentum $\boldsymbol{K}$ have helicity $\chi=+1$ and 
$\chi=-1$ respectively. Moreover, under the action of the effective magnetic field these states of opposite helicity are energetically split. The non-zero helicity and the effective Zeeman splitting of the $F_{z_{B}}= \pm 1$ states in an orthorhombic system gives rise to CD.

A Pure 2D Rashba

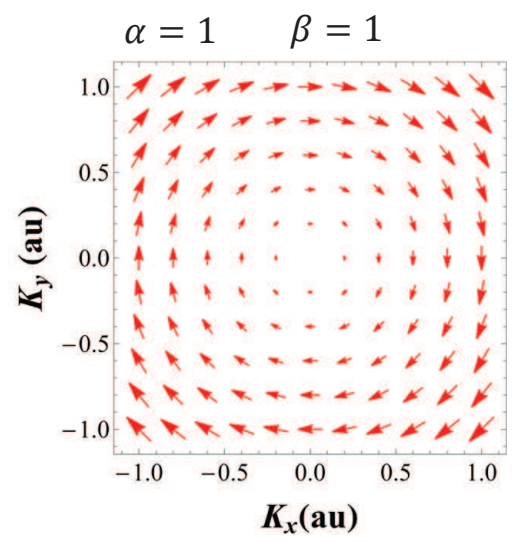

C Mixed Rashba/Dresselhaus

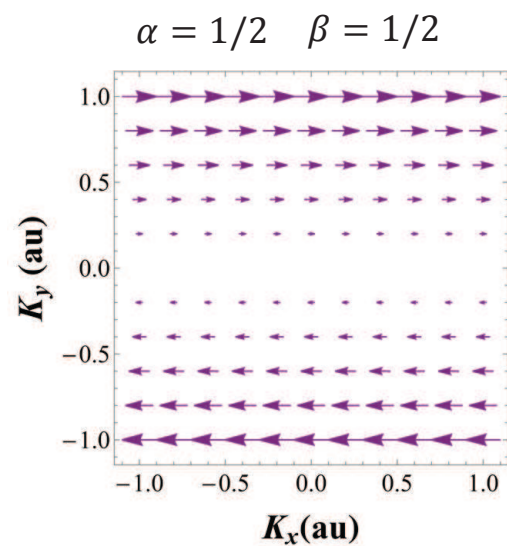

B Pure 2D Dresselhaus

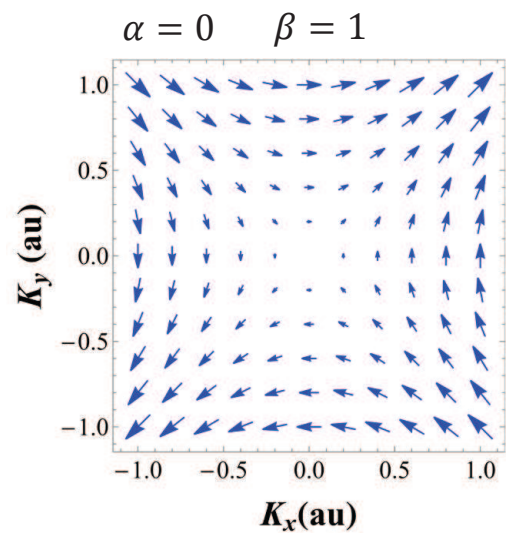

D Mixed Rashba/Dresselhaus

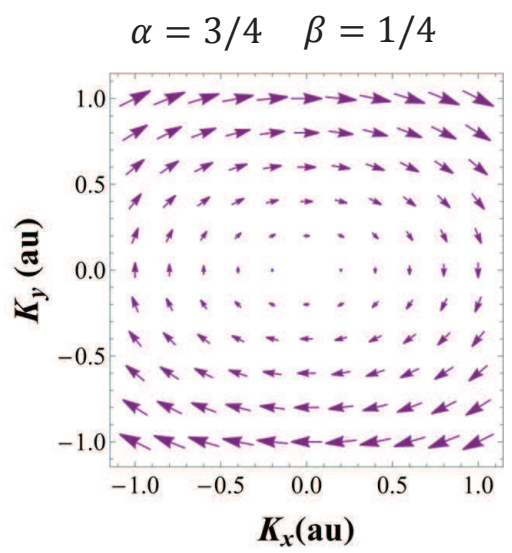

Figure 2. Vector field maps of the effective magnetic field due to exciton center of mass motion in the presence of Rashba splitting. The figure shows the relative magnitude and direction of the effective magnetic field defined in Eq. 10 in the $K_{x}, K_{y}$ plane. The $K_{x}$ and $K_{y}$ axes are plotted in arbitrary units (au) in the figure. Panel A shows the relative effective magnetic field map for the pure Rashba case, ( $\alpha=1, \beta=0$ in dimensionless units), while panel $\mathrm{B}$ shows the pure 2D Dresselhaus case ( $\alpha=0, \beta=1$ ). Panels $\mathrm{C}$ and $\mathrm{D}$ show mixed cases corresponding respectively to dimensionless $\alpha=\beta=1 / 2$, and $\alpha=3 / 4, \beta=1 / 4$. For each case the components of the Rashba tensor $\alpha_{x y}$ and $\alpha_{y x}$ corresponding to Eq. [6 are also given.

We proceed now to a quantitative analysis of the effect of the Rashba terms on the exciton fine structure and dichroic optical properties. The exciton Rashba Hamiltonian described by 
Eq. [6 for non-zero $\boldsymbol{K}$ splits the degeneracy of the 4 exciton states. Taking into account the exciton kinetic energy $\hbar^{2} K^{2} / 2 M$, where $K=\sqrt{K_{x}^{2}+K_{y}^{2}}$, the energy for any momentum direction is determined as a function of $K_{x}, K_{y}$ by (see the Supporting Information),

$$
E_{C O M}(K)=\frac{\hbar^{2} K^{2}}{2 M} \pm \frac{m_{e}}{M} \sqrt{\alpha_{y x}^{2} K_{x}^{2}+\alpha_{x y}^{2} K_{y}^{2}}
$$

The COM motion of free excitons described by Eq. (11) has the offset parabolic dispersion, well known for $2 \mathrm{D}$ electrons in the presence of Rashba terms. $\underline{44}$

A complete description of the exciton dispersion requires, however, that we also include the exciton fine structure splitting due to electron-hole exchange, resulting in the following Hamiltonian:

$$
\hat{H}_{\mathrm{tot}}=\hat{H}_{\mathrm{INT}}+\hat{H}_{R, \mathrm{COM}}^{D, X, Y, Z}(\boldsymbol{K})
$$

where $\hat{H}_{\text {INT }}$ describes the fine structure of the exciton connected with exciton internal motion at $K=0$. It is straightforward to write $\hat{H}_{\text {tot }}$ in a basis of the four electron and hole Bloch function products, $\left|u_{j_{e}=1 / 2}\right\rangle\left|u_{j_{h}=1 / 2}\right\rangle,\left|u_{j_{e}=1 / 2}\right\rangle\left|u_{j_{h}=-1 / 2}\right\rangle,\left|u_{j_{e}=-1 / 2}\right\rangle\left|u_{j_{h}=1 / 2}\right\rangle$ and $\left|u_{j_{e}=-1 / 2}\right\rangle\left|u_{j_{h}=-1 / 2}\right\rangle$. To study the exciton polarization properties it is most convenient to transform into a basis of exciton states whose dipoles are oriented along the $x, y, z$ directions. ${ }^{45}$ This $\mathcal{O}$ basis is described by the $|X\rangle,|Y\rangle$, and $|Z\rangle$ wave functions of the three exciton states $X, Y$, and $Z$ whose dipoles are oriented along the $x, y$, and $z$ directions, and the $|D\rangle$ wave function of the dark $D$ exciton state, which is dipole inactive. ${ }^{45}$ The unitary transformation to this basis, developed in the Supporting Information section, results in the following matrix representation,

$$
\begin{aligned}
\hat{H}_{D, X, Y, Z}^{\mathrm{tot}}(\boldsymbol{K})= & {\left[\mathcal{E}_{0,0}+\frac{\hbar^{2}\left(K_{x}^{2}+K_{y}^{2}\right.}{2 M}\right] \mathbb{I}+} \\
& \left(\begin{array}{cccc}
E_{d} & -\alpha_{x y} K_{y} & \alpha_{y x} K_{x} & 0 \\
-\alpha_{x y} K_{y} & E_{x} & 0 & -i \alpha_{y x} K_{x} \\
\alpha_{y x} K_{x} & 0 & E_{y} & -i \alpha_{x y} K_{y} \\
0 & i \alpha_{y x} K_{x} & i \alpha_{x y} K_{y} & E_{z}
\end{array}\right),
\end{aligned}
$$

where $\mathbb{I}$ is the $4 \times 4$ unit matrix. The second term of Hamiltonian $\hat{H}_{D, X, Y, Z}^{\text {tot }}(\boldsymbol{K})$ describes the exciton fine structure, where $E_{d}$ is energy of the dark exciton at $\boldsymbol{K}=0$, while the bright triplet states $X, Y$, and $Z$ have $\boldsymbol{K}=0$ energies $E_{x}, E_{y}$, and $E_{z}$ respectively. These are degenerate in the case of cubic symmetry but split in general in crystals with orthorhombic 
crystal structure. $\stackrel{42}{45}$ In our calculations, for simplicity, we will start by neglecting the crystal field splitting between the $X, Y$ and $Z$ exciton states, setting $E_{x}=E_{y}=E_{z}=E_{t}$, the bright triplet energy. Although the off-diagonal terms in Hamiltonian described by Eq. (13) generally are not rotationally invariant, we have found closed form solutions for the eigenvalues of $\hat{H}_{D, X, Y, Z}^{\text {tot }}(\boldsymbol{K})$ in several special cases. In the case that crystal field splitting can be neglected, $E_{x}=E_{y}=E_{z}=E_{t}$, assuming that $\alpha_{y x}=\alpha_{x y}$ (the pure Rashba case $\alpha_{x y}=\alpha$ ), or $\alpha_{y x}=-\alpha_{x y}$ (the pure Dresselhaus case, $\alpha_{x y}=\beta$ ) we obtain,

$$
\begin{aligned}
E_{1, \pm 1}(\boldsymbol{K}) & =\mathcal{E}_{0,0}+\Delta+\frac{\hbar^{2} K^{2}}{2 M} \pm K \frac{m_{e} \alpha_{x y}}{M} \\
E_{1 / 2 \pm 1 / 2,0}(\boldsymbol{K}) & =\mathcal{E}_{0,0}+\frac{\hbar^{2} K^{2}}{2 M}+\frac{\Delta}{2} \pm \frac{\sqrt{\Delta^{2} M^{2}+4 K^{2}\left(m_{e} \alpha_{x y}\right)^{2}}}{2 M} .
\end{aligned}
$$

Here, $\Delta \equiv E_{t}-E_{d}$ is the energy difference between the bright triplet states and the dark exciton state. The subscripts on the energy in this expression identify the projection of the exciton angular momentum, $F_{z_{B}}$ along the quantization axis $\boldsymbol{z}_{B}$ taken in the direction of the effective magnetic field $\boldsymbol{B}^{\text {eff }}(\boldsymbol{K})$. The direction $\boldsymbol{z}_{B}$ thus varies with $\boldsymbol{K}$ as described in Eq. 10 and in Fig. 2. The analytical form for the exciton dispersion can also be found in the case that the triplet degeneracy at $\boldsymbol{K}=0$ is lifted, with $E_{z} \neq E_{t}$ (see SI). Generally, however, for any symmetry, the fine structure of the ground exciton state can be written as $E_{j}(\boldsymbol{K})=E_{1,0}^{0}(K)+\delta_{j}(\boldsymbol{K})$, where $E_{1,0}^{0}(K)$ from Eq. 3 is the exciton energy in the absence of fine structure splitting $\delta_{j}(\boldsymbol{K})$. The latter is found by diagonalization of Hamiltonian $\hat{H}_{\text {tot }}$ from Eq. (12) (for details see SI).

$$
\Psi_{\boldsymbol{K}, j}(\boldsymbol{R}, \boldsymbol{r})=\frac{\exp (i \boldsymbol{K} \cdot \boldsymbol{R})}{\sqrt{S}} \phi_{1,0}(\boldsymbol{r}) \sum_{i=D, X, Y, Z} C_{j_{e}, j_{h}}^{j}(\boldsymbol{K})\left|u_{i}\right\rangle
$$

where the coefficients $C_{i}^{j}(\boldsymbol{K})$ describe the eigenstate of the corresponding matrix in the basis of exciton states $|D\rangle,|X\rangle,|Y\rangle,|Z\rangle$.

Figure 3 shows the exciton energies calculated along the $K_{y}$ direction using Eq. 14, Panels A and B show the energies calculated for pure 2D-Rashba and pure 2D-Dresselhaus spin textures, with $\alpha=156 \mathrm{meV} \cdot \mathrm{nm}$ and $\beta=156 \mathrm{meV} \cdot \mathrm{nm}$, respectively, for electron and hole effective masses $m_{e}=m_{h}=0.25 m_{0}$, where $m_{0}$ is the free electron mass. These parameters each correspond to a Rashba energy of $40 \mathrm{meV}$ as measured in the 2D HOIS, PEPI. ${ }^{35}$ The calculations shown assume degenerate triplet levels at $\boldsymbol{K}=0$ at energy $\Delta=8$ meV above the dark state at energy $E_{D}=0 \mathrm{meV}$, corresponding to the exchange constant 
$w=12$ meV measured in the $2 \mathrm{D}$ hybrid organic $\left(\mathrm{C}_{6} \mathrm{H}_{13} \mathrm{NH}_{3}\right)_{2} \mathrm{PbI}_{4} \stackrel{46}{\underline{4}}$ The dispersion curves for each level are labelled according to $F, F_{z_{B}}$, the exciton total angular momentum and its projection on an axis aligned to the $\boldsymbol{K}$ dependent effective magnetic field $\boldsymbol{B}^{e f f}(\boldsymbol{K})$, see Eq. 10 and Figure 2 which shows the field direction in the $K_{x}, K_{y}$ plane. Referring to Fig. 2, we call attention in particular to the fact that the direction of $B^{e f f}$ reverses in passing through the origin; with our state labelling convention, the $F_{z_{B}}=+1$ state always has higher energy than the one with $F_{z_{B}}=-1$. The dispersion in $\mathrm{A}$ and $\mathrm{B}$ are identical reflecting isotropy about the $\hat{z}$ axis. This symmetry is evident in the insets of each figure, which show 3D plots of the energy surfaces in the $K_{x}, K_{y}$ plane for the levels $\left(F, F_{z}\right)=(1, \pm 1)$. Panels $\mathrm{C}$ and $\mathrm{D}$ show the angular momentum textures for these two states along constant energy contours plotted at $E=70 \mathrm{meV}$, chosen simply for clarity of the display. The direction of the effective magnetic field depends differently on $K_{x}, K_{y}$ for the pure Rashba versus the pure Dresselhaus spin textures (see Figure 2), resulting in the distinctly different exciton angular momentum textures shown in panels $\mathrm{C}$ and $\mathrm{D}$. The $F_{z_{B}}= \pm 1$ exciton states for the pure Dresselhaus case, panel D, exhibit helicity for any wave vector not aligned to the mirror plane directions $\hat{x}$ and $\hat{y}$, with maximum helicity realized along the lines $K_{x}= \pm K_{y}$. The corresponding exciton states for the pure Rashba case are achiral since the angular momentum is orthogonal to $\boldsymbol{K}$ for all $\boldsymbol{K}$.

The significance of the helicity consideration is underscored by examination of the exciton eigenvectors, transition dipoles and helicity corresponding to Fig. Table I shows these for the case $E_{z}=E_{t}$ for both the Rashba angular momentum texture and the pure 2D Dresselhaus angular momentum texture, respectively. The energy eigenvalues are given by Eq. 14 along the line $K_{y}=K_{x}$ for positive $K_{x}$, using $\alpha=\alpha_{x y}=\alpha_{y x}$ for the Rashba case, and $\beta=\alpha_{x y}=-\alpha_{y x}$ for the Dresselhaus case. The table shows the expansion coefficients of the exciton wavefunction in the $|D\rangle,\left|X^{\prime}\right\rangle,\left|Y^{\prime}\right\rangle$, and $|Z\rangle$ basis, which is rotated so that the $x^{\prime}$ axis is aligned with $\boldsymbol{K}$. The polarization properties of each exciton state is shown in the last column, also given in the rotated coordinate system. While there are two transition dipoles in the Rashba case (for the $F_{z_{B}}= \pm 1$ states) that appear circular $\left(\hat{x}^{\prime} \pm i \hat{z}\right)$, they are not actually helical because the components of the transition dipoles parallel to the $x^{\prime}$ direction cannot couple to transverse electro-magnetic waves propagating in the $x^{\prime}$ direction. These results actually hold for the Rashba case for any wave vector $\boldsymbol{K}$ due to the rotational invariance of Eq. [6] when $\alpha_{x y}=\alpha_{y x}$. For the pure Dresselhaus angular 

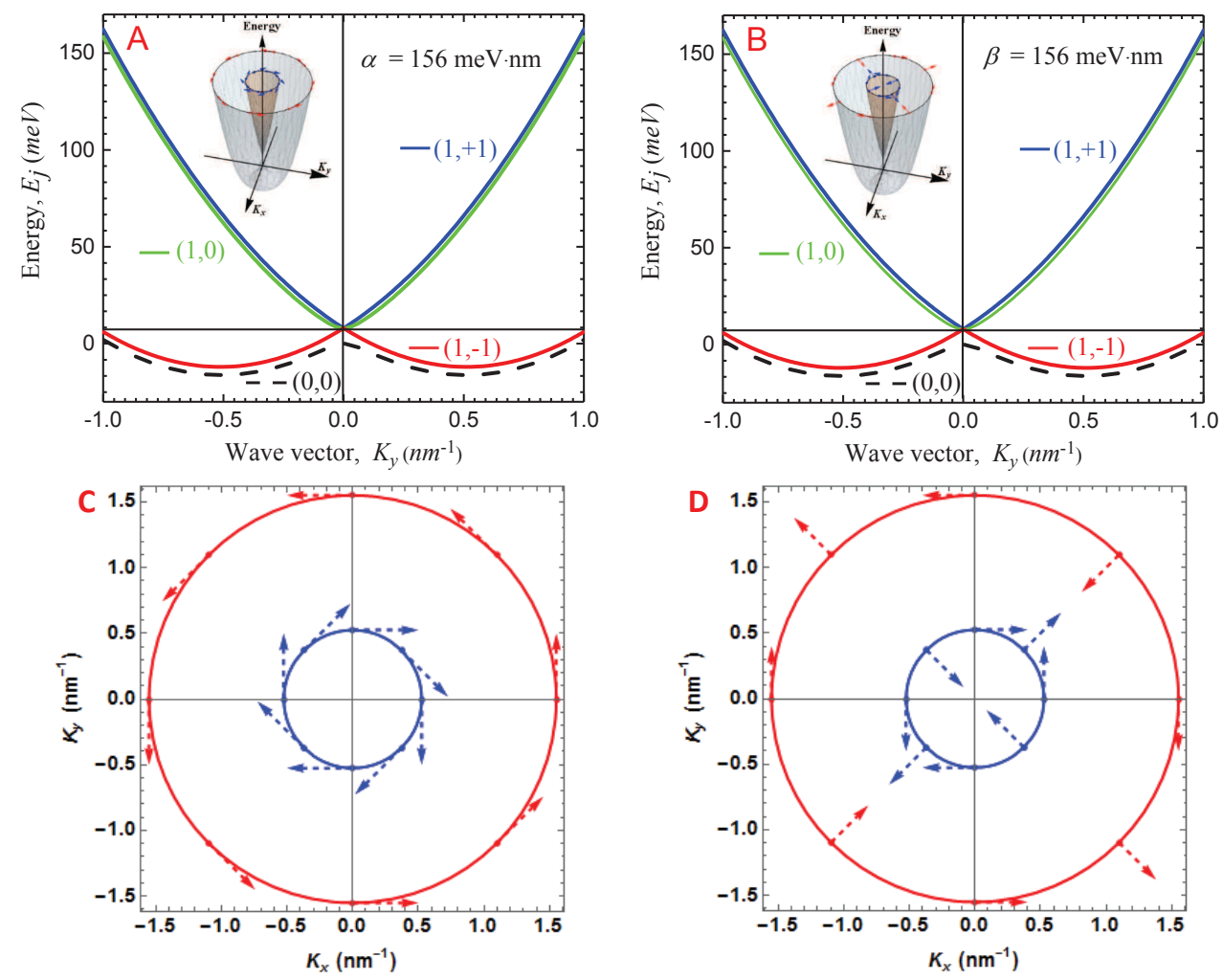

Figure 3. Exciton energies and angular momentum textures in 2D layered perovskite with inversion symmetry breaking along the $\hat{z}$ direction normal to the $2 \mathrm{D}$ plane. Panels $\mathrm{A}$ and $\mathrm{B}$ show the energies calculated versus wave vector $\boldsymbol{K}=K_{y} \hat{\boldsymbol{y}}$ for pure Rashba and pure 2D-Dresselhaus angular momentum textures, respectively, with $\alpha=156 \mathrm{meV} \cdot \mathrm{nm}$ and $\beta=156 \mathrm{meV} \cdot \mathrm{nm}$ with electron and hole effective masses $m_{e}=m_{h}=0.25 m_{0}$, and assuming degenerate triplet levels at $\boldsymbol{K}=0$ at energy $\Delta=8 \mathrm{meV}$ above the dark state at energy $E_{D}=0 \mathrm{meV} \underline{\underline{46}}$ The dispersion curves for each fine structure level in panels $\mathrm{A}$ and $\mathrm{B}$ are labelled according to $\left(F, F_{z_{B}}\right)$, the exciton total angular momentum and its projection on an axis aligned to the effective magnetic field, see Eq,10 and Figure 2, The curves in A and B are identical reflecting symmetry under rotation about the $\hat{z}$ axis as evident in the insets of each figure, which show 3D plots of the energy surfaces in the $K_{x}, K_{y}$ plane for the levels $(1, \pm 1)$. Panels $\mathrm{C}$ and $\mathrm{D}$ show the angular momentum textures for these two states along constant energy contours at $E=70 \mathrm{meV}$.

momentum texture however, it is the $Y^{\prime}$ and $Z$ states that are mixed with each other with a relative phase factor of $i$. Since these dipole components are both normal to the direction of propagation $\boldsymbol{K}$ along the $x^{\prime}$ direction, these states have circularly polarized transition 
dipoles. Since they are split, these transitions exhibit CD as discussed above in the context of the effective magnetic field description.

This consideration shows that the magnitude of CD depends on the exciton propagation angle $\phi$ relative to the mirror symmetry planes. Indeed, if this system were to be side illuminated along the $\phi=45^{\circ}$ azimuth the $F_{Z_{B}}= \pm 1$ states would exhibit $100 \%$ degree of circular polarization. In Fig. 4 we show the angular dependence of the polarization structure of the exciton sublevels calculated taking into account both Rashba and Dresselhaus terms as well as the exciton fine structure connected with exciton internal motion.

\section{CALCULATION OF CIRCULAR DICHROISM}

The analysis conducted in the previous section showed that both cubic and tetragonal symmetry are too high to observe circular dichroism connected with exciton COM motion at zero magnetic field. Intrinsic circular dichroism in perovskites requires orthorhombic symmetry, which is required to realize 2D Dresselhaus angular momentum textures. To describe the CD, let us consider the probability of excitation of $2 \mathrm{D}$ excitons by absorption of light. We consider the ground state of the exciton in the $x, y$ plane of the 2D layer created by absorption of light incident on the 2 D layer with wave vector $\boldsymbol{k}_{p h}$ at angle $\theta$ measured from the vertical direction $\hat{\boldsymbol{n}}_{z}$ (Fig 1).

The light interacting with the 2D perovskite layer, may be absorbed to create an exciton. The probability of excitation of the $j^{\text {th }}$ exciton sub-level of the ground $2 \mathrm{D}$ exciton state with in-plane wave vector $\boldsymbol{K}$ can be described by Fermi's golden rule:

$$
\mathcal{W}_{\boldsymbol{K}, j}=\frac{2 \pi}{\hbar}\left|\left\langle\Psi_{\boldsymbol{K}, j}\left|\hat{H}_{\mathrm{int}}\right| G\right\rangle\right|^{2} \delta\left(E_{j}(\boldsymbol{K})-\hbar \omega\right) .
$$

Here, $|G\rangle$ is the crystal ground state, $\hbar \omega$ is the energy of the absorbed photon, and $E_{j}(\boldsymbol{K})$ is the energy of the $j^{\text {th }}$ exciton sub-level. The light-matter interaction Hamiltonian $\hat{H}_{\text {int }}=$ $-\left(e / m_{0} c\right) \boldsymbol{A}^{m} \cdot \hat{\boldsymbol{p}}$ is expressed as usual in terms of the inner product of the dipole operator $\hat{\boldsymbol{p}}$ and the vector potential $\boldsymbol{A}^{m}$ inside the 2D layer. Evaluating the matrix element in Eq. 16 for the exciton state $\Psi_{\boldsymbol{K}, j}\left(\boldsymbol{r}_{e}, \boldsymbol{r}_{h}\right)$ from Eq. (15) we find,

$$
\left\langle\Psi_{\boldsymbol{K}, j}\left|\hat{H}_{\mathrm{int}}\right| G\right\rangle=\frac{e}{m_{0} c} \boldsymbol{A}^{m} \cdot \boldsymbol{P}_{j}(\boldsymbol{K}) \phi_{1,0}(0) \delta_{\boldsymbol{k}_{p h, \|}, \boldsymbol{K}}
$$

The Kronecker delta represents the well-known momentum conservation rule, reflecting conservation of momentum in the $2 \mathrm{D}$ layer. The wave vector $\boldsymbol{K}$ of the in-plane exciton created 


\begin{tabular}{|c|c|c|c|}
\hline \multicolumn{4}{|c|}{ Pure 2D Rashba, $\alpha=\alpha_{x y}=\alpha_{y x}$} \\
\hline Energies & $\left\{D, X^{\prime}, Y^{\prime}, Z\right\}$ & Polarization, $\boldsymbol{P}_{j} / P_{j}$ & Helicity $\chi$ \\
\hline$E_{1,+1}(\boldsymbol{K})$ & $\frac{1}{\sqrt{2}}\{0,1,0,+i\}$ & $\frac{1}{\sqrt{2}}\left(\hat{x}^{\prime}-i \hat{z}\right)$ & 0 \\
$E_{1,-1}(\boldsymbol{K})$ & $\frac{1}{\sqrt{2}}\{0,1,0,-i\}$ & 0 \\
$E_{1,0}(\boldsymbol{K})$ & $\frac{1}{\sqrt{N_{1,0}}}\left\{-\frac{\Delta M-\sqrt{\Delta^{2} M^{2}+4 K^{2}\left(m_{e} \alpha\right)^{2}}}{2 K m_{e} \alpha}, 0,1,0\right\}$ & $\hat{y}^{\prime}$ & 0 \\
$E_{0,0}(\boldsymbol{K})$ & $\frac{1}{\sqrt{N_{0,0}}}\left\{\frac{\Delta M-\sqrt{\Delta^{2} M^{2}+4 K^{2}\left(m_{e} \alpha\right)^{2}}}{2 K m_{e} \alpha}, 0,1,0\right\}$ & $\hat{y}^{\prime}$ & 0 \\
\hline & \multicolumn{2}{|c|}{ Pure $2 \mathrm{D}$ Dresselhaus, $\beta=\alpha_{x y}=-\alpha_{y x}$} & +1 \\
\hline$E_{1,+1}(\boldsymbol{K})$ & $\frac{1}{\sqrt{2}}\{0,0,1,+i\}$ & $\frac{1}{\sqrt{2}}\left(\hat{y}^{\prime}-i \hat{z}\right)$ & -1 \\
$E_{1,-1}(\boldsymbol{K})$ & $\frac{1}{\sqrt{2}}\{0,0,1,-i\}$ & $\frac{1}{\sqrt{2}}\left(\hat{y}^{\prime}+i \hat{z}\right)$ & 0 \\
$E_{1,0}(\boldsymbol{K})$ & $\frac{1}{\sqrt{N_{1,0}}}\left\{\frac{\Delta M-\sqrt{\Delta^{2} M^{2}+4 K^{2}\left(m_{e} \beta\right)^{2}}}{2 K m_{e} \beta}, 1,0,0\right\}$ & $x^{\prime}$ & 0 \\
$E_{0,0}(\boldsymbol{K})$ & $\frac{1}{\sqrt{N_{0,0}}}\left\{-\frac{\Delta M-\sqrt{\Delta^{2} M^{2}+4 K^{2}\left(m_{e} \beta\right)^{2}}}{2 K m_{e} \beta}, 1,0,0\right\}$ & $x^{\prime}$ & +1 \\
\hline
\end{tabular}

Table I. Energies, wave functions, polarizations and helicity of exciton states with wave vector $\boldsymbol{K}$ along the line $K_{y}=K_{x}$ for positive $K_{x}$, for the pure 2D Rashba spin-texture, top, and the pure 2D Dresselhaus spin texture, bottom. Energies labelled according to the total angular momentum $F$ and its projection along the effective magnetic field direction, $F_{z_{B}}$, and are given for each state by Eq. 14. For the Rashba case, $\alpha=\alpha_{x y}=\alpha_{y x}$, while for the Dresselhaus case, $\beta=\alpha_{x y}=-\alpha_{y x}$ in Eq. 11, Results are shown neglecting the crystal field splitting between the $Z$ exciton and the $X$ and $Y$ excitons $\left(E_{z}=E_{t}\right)$. The table shows the energy and the expansion coefficients of the exciton wavefunction in the $|D\rangle,\left|X^{\prime}\right\rangle,\left|Y^{\prime}\right\rangle$, and $|Z\rangle$ basis (see Eq, 15), which is rotated so that the $x^{\prime}$ axis is aligned with $\boldsymbol{K}$. The factors $N_{1,0}$ and $N_{0,0}$ are normalization coefficients. The polarization properties of each exciton state are given in the rotated coordinate system. The last column gives the helicity, $\chi=\boldsymbol{F} \cdot \hat{\boldsymbol{n}}_{\boldsymbol{K}}$, for each state.

by absorption of a photon must match the in-plane component $\boldsymbol{k}_{p h, \|}=\boldsymbol{k}_{p h}-\hat{\boldsymbol{n}}_{z}\left(\boldsymbol{k}_{p h} \cdot \hat{\boldsymbol{n}}_{z}\right)$, of the photon that was absorbed, and has magntitude $k_{p h} \sin \theta$. The contact term $\phi_{n, 0}(0) \neq$ 0 in Eq. 17 only for states with internal motion azimuthal quantum number $m=0$ ("s"states).

The key factor in Eq 17 that is responsible for the polarization properties of absorption is the dipole transition matrix element for the exciton. In terms of the $|D\rangle,|X\rangle,|Y\rangle$, and 
$|Z\rangle$ exciton basis, we can express the exciton transition dipole matrix element as,

$$
\boldsymbol{P}_{j}(\boldsymbol{K})=\sum_{i=x, y, z}\left[C_{i}^{j}(\boldsymbol{K})\right]^{*} \boldsymbol{P}_{i}=P_{c v} \sum_{i=x, y, z}\left[C_{i}^{j}(\boldsymbol{K})\right]^{*} g_{i} \hat{\boldsymbol{n}}_{i}
$$

where $\hat{\boldsymbol{n}}_{i}$ are the unit vectors along the $x, y$ and $z$ directions, $P_{c v}=-i\left\langle S\left|\hat{\boldsymbol{p}}_{z}\right| z\right\rangle$ is the Kane momentum matrix element, assumed equal for the three p-like conduction band states, $|x\rangle,|y\rangle$ and $|z\rangle$, while $g_{i}$ is a dimensionless parameter giving the relative magnitudes of the transition dipole matrix elements of the $|X\rangle,|Y\rangle$ and $|Z\rangle$ exciton basis states. In our calculations we assumed that the $X, Y$, and $Z$ exciton basis states have transition dipoles of equal relative magnitude, $g_{x}=g_{y}=g_{z}$. (Expressions for $g_{i}$ accounting for crystal field effects within a six-band $\boldsymbol{K} \cdot \boldsymbol{P}$ model can be found in Ref. 45 ).

To find the absorption coefficient we need to find vector potential of light $\boldsymbol{A}^{m}$ inside the perovskite. We first consider the incident light to be circularly polarized in air with helicity \pm 1 , corresponding to angular momentum \pm 1 along the $\boldsymbol{k}_{p h}$ direction. However, accounting for refraction, the angle of propagation of the light in the perovskite, $\theta_{\text {mat }}$, is modified from the exterior angle of incidence $\theta$. Neglecting linear birefringence effects for simplicity, this is given by Snell's law, $\sin \theta=n_{\text {mat }} \sin \theta_{\text {mat }}$. Moreover the amplitude of the transmitted electric field or vector potential is modified by the polarization-dependent Fresnel amplitude transmission coefficients $t_{\|}$and $t_{\perp}$ for field components parallel and perpendicular to the plane of incidence, respectively, which are shown in Figure S3 in the Supporting Information.

At large incidence angles $\theta$ the degree of circular polarization of the transmitted light is reduced because the field component perpendicular to the plane of incidence has lower transmission that the field parallel component. This effect needs to be taken into account since the polarization of the incident circular light is set before refraction into the sample. For circular light with helicity $\hat{e}_{ \pm}$propagating as shown in the inset of Fig. 4, the light transmitted into the sample has amplitude (see the Supporting Information section),

$$
\boldsymbol{A}_{ \pm}^{m}\left(\theta_{m a t}, \phi\right)=A_{0}\left(t_{\|}\left(\theta_{m a t}\right) \hat{e}_{\|}\left(\theta_{m a t}, \phi\right) \pm i t_{\perp}\left(\theta_{m a t}\right) \hat{e}_{\perp}\left(\theta_{m a t}, \phi\right)\right)
$$

Using these expressions we can write the strength of interaction of the exciton state $j$ with the vector potential $\boldsymbol{A}_{ \pm}^{m}\left(\theta_{m a t}, \phi\right)$, dropping common factors, as,

$$
I^{ \pm}(j)=\left|\boldsymbol{A}_{ \pm}^{m}\left(\theta_{m a t}, \phi\right) \cdot \boldsymbol{P}_{j}(\boldsymbol{K})\right|^{2}
$$

It is useful to define a normalized interaction strength for a given set of angles. This should be normalized by the square of the magnitude of the light field, $\left|A_{ \pm}^{m}\left(\theta_{\text {mat }}, \phi\right)\right|^{2}$. As discussed 
in the Supporting information section, we normalize the magnitude of the transition dipole of each exciton state to the average norm-squared dipoles of the $X, Y, Z$ basis states, which we denote as $\tilde{f}_{N}\left|P_{c v}\right|^{2}$, where $\tilde{f}_{N}=\left(g_{x}^{2}+g_{y}^{2}+g_{z}^{2}\right) / 3$. Then the normalized light-matter interaction strength for exciton state $\boldsymbol{K}, j$ is,

$$
I_{N}^{ \pm}(j)=\frac{\left|\boldsymbol{A}_{ \pm}^{m}\left(\theta_{\text {mat }}, \phi\right) \cdot \boldsymbol{P}_{j}(\boldsymbol{K})\right|^{2}}{\left|A_{ \pm}^{m}\left(\theta_{\text {mat }}, \phi\right)\right|^{2} \tilde{f}_{N}\left|P_{c v}\right|^{2}}
$$

With this definition we define the magnitude of circular dichroism for the state $j$ as,

$$
\mathcal{P}_{j}=I_{N}^{+}(j)-I_{N}^{-}(j)
$$

We note that this definition coincides with the degree of polarization for the exciton state in a cubic perovskite with total angular momentum $F_{z}= \pm 1$ interacting with circularly polarized light propagating in the $+\hat{z}$ direction.

We consider the sample, depicted in Fig. \$ A, to be oriented in the $x, y$ plane, with the 2D layers parallel to the top surface of the sample which is normal to the $z$ direction. The sample has two-fold rotational symmetry about the $z$ axis, with inversion symmetry broken along the $+\hat{z}$ direction, and mirror symmetry through the $x, z$ and $y, z$ planes. The sample is assumed to have refractive index $n_{\text {mat }}$ and to be illuminated from air at a polar incidence angle $\theta$ measured from the vertical $z$ axis, in a plane of incidence defined by azimuthal angle $\phi$ measured from $x$.

In Figure 4 we show plots of the fine structure energy splitting and the CD signal for various light incidence geometries and sample parameters. In the figure, the refractive index of the perovskite is assumed to be $n=\sqrt{5}$ and the wavelength of light resonant with the exciton transition is taken as $\lambda_{0}=500 \mathrm{~nm}$ in free space. The schematic in Fig 4 panel A shows circularly polarized light incident at polar angle $\theta$ measured from the layer normal, at azimuth angle $\phi$ measured from the $\hat{x}$ symmetry axis. As the polar angle increases, the exciton COM momentum increases, as shown in panel B. The exchange constant used in the calculations, taken as $w=12 \mathrm{meV}$, reflects the measured value in the $2 \mathrm{D}$ hybrid organic

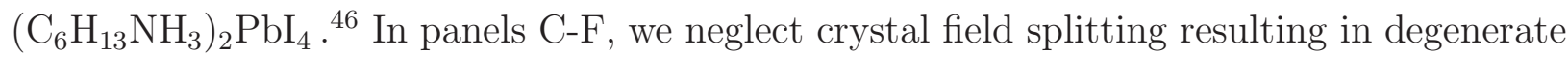
triplet exciton levels at $\boldsymbol{K}=0$ at energy $E_{t}=8 \mathrm{meV}$ above the dark state at energy $E_{D}=0 \mathrm{meV}$ as in Fig. 3. Panels $\mathrm{C}$ and E show the CD signal, Eq. 22, calculated for each fine structure level versus the azimuth angle for a fixed polar angle $\theta$ of $45^{\circ}$, while panels $\mathrm{D}$ and $\mathrm{F}$ show the $\mathrm{CD}$ versus the polar angle for a fixed azimuth angle of $45^{\circ}$. The upper 

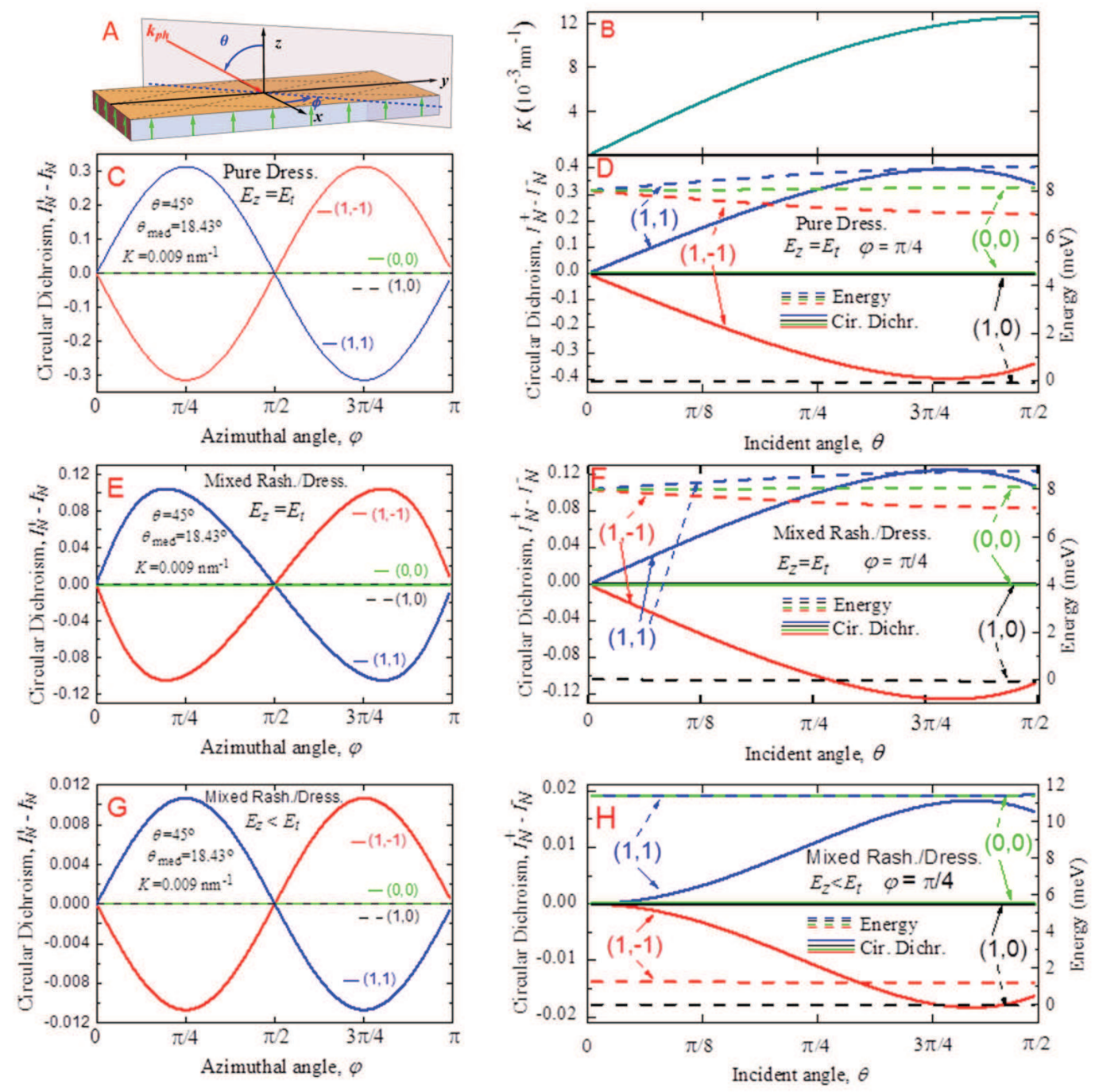

Figure 4. CD signal versus light incidence geometry in a 2D perovskite with degenerate triplet states at $\boldsymbol{K}=0$. The schematic in A shows circularly polarized light incident at polar angle $\theta$ measured from the layer normal, at azimuth angle $\phi$ measured from the $x$ axis. The effective masses are $m_{e}=m_{h}=0.25 m_{0}$ throughout. In panels C-F, calculations are performed assuming degenerate triplet levels at $\boldsymbol{K}=0$ at energy $\Delta=8 \mathrm{meV}$ above the dark state at energy $E_{D}=$ $0 \mathrm{meV}$ as in Fig. 3. Panels $\mathrm{C}$ and $\mathrm{E}$ show the $\mathrm{CD}$ for fine structure level versus $\phi$ for a fixed polar angle $\theta=45^{\circ}$, while panels $\mathrm{D}$ and $\mathrm{F}$ show the $\mathrm{CD}$ versus $\theta$ for fixed $\phi=45^{\circ}$. Panels $\mathrm{C}$, D reflect pure Dresselhaus spin texture with $\alpha=0$ and $\beta=156 \mathrm{meV} \cdot \mathrm{nm}$, while panels E,F are calculated for mixed Rashba/Dresselhaus spin textures with parameters with $\alpha=117 \mathrm{meV} \cdot \mathrm{nm}$ and $\beta=39 \mathrm{meV} \cdot \mathrm{nm}$. Panels $\mathrm{G}-\mathrm{H}$ are calculated as in E,F, but with crystal field splitting of the bright triplet, reflected in $E_{t}=11.4 \mathrm{meV}, E_{z}=1.2 \mathrm{meV}$. 
two panels, (C,D) are calculated for pure Dresselhaus spin texture with $\alpha=0$ and $\beta=$ $156 \mathrm{meV} \cdot \mathrm{nm}$, corresponding to a Rashba energy for the conduction band $E_{R}=40$ meV following Vardeny, et al., Ref. 35. The middle panels (E,F) are calculated for mixed Rashba/Dresselhaus spin textures with parameters with $\alpha=117 \mathrm{meV} \cdot \mathrm{nm}$ and $\beta=$ $39 \mathrm{meV} \cdot \mathrm{nm}$, and show a reduction in the CD relative to the pure Dresselhaus case as expected. The maximum CD in the pure Dresselhaus case occurs along azimuths at 45 degrees from the mirror planes as expected, while for the mixed case the max CD azimuths are slightly shifted reflecting warping of the constant energy contours for angular momentum textures of mixed character. Panels C,D,E show that the CD generally increases with polar angle $\theta$ as the in-plane exciton momentum increases; the fall off as $\theta$ approaches 90 degrees reflects the decreasing degree of circular polarization of the transmitted light described in Eq. 19 as the polar angle increases. The maximum CD signal is primarily limited by the angle of propagation of light in the material, $\theta_{\text {mat }}$, given the assumption that illumination is from the top surface of the 2D layer sample. Under conditions of side illumination, the CD signals in Fig. 4 would be significantly larger; the maximum CD signal for $\boldsymbol{k}_{p h}$ side incident along the $\hat{x}+\hat{y}$ direction for the material parameters reflected in panel $\mathrm{D}$ would be \pm 1 for the $F_{z_{B}}= \pm 1$ exciton sublevels.

In panels $\mathrm{G}-\mathrm{H}$ of Fig 4 we repeat the calculations shown in panels E-F, but now take account of the effect of crystal field splitting. Following the measurements of Refs. $\underline{46}, \underline{47}$ we set the upper bright doublet at $E_{x}=E_{y}=11.4 \mathrm{meV}$ and the lower bright singlet $E_{z}=1.2 \mathrm{meV}$ above the dark singlet at energy $0 \mathrm{meV}$. Since the CD signal results from mixing of the $Z$ and the $Y^{\prime}$ exciton states under the action of the effective magnetic field as described above, additional fine structure splitting between these states causes a reduction in the CD signal by over an order of magnitude.

The analysis so far provides a set of definitions for describing the CD of an individual exciton fine structure level $j$. In practice, the exciton transitions are spectrally broadened so that the individual exciton sublevel transitions may not be individually resolvable. We model the effect of linewidth broadening by convolving the absorption spectra with a Gaussian lineshape function,

$$
G(E)=\frac{1}{\sqrt{2 \pi \sigma}} e^{-E^{2} /\left(2 \sigma^{2}\right)}
$$

where the full-width at half maximum linewidth (LW) is given by $2 \sqrt{2 \ln 2} \sigma$. We then 
calculate a normalized absorption spectrum for plus and minus circularly polarized incident light as a function of energy, $I_{N}^{ \pm}(E)$, parametrically as a function of the angles $\theta$ and $\phi$ :

$$
I_{N}^{ \pm}(E)=\frac{1}{I_{\max }} \sum_{j} I_{N}^{ \pm}(j) G\left(E-E_{j}\right) .
$$

In this expression, the sum is taken over the four exciton sub-levels $j$ for a given $\boldsymbol{K}$, and a normalization factor $I_{\max }$ is included to normalize the peak absorption to unity. Then the spectral CD signal is calculated as the difference of the normalized absorption spectra for positive and negative circularly polarized light:

$$
P(E)=I_{N}^{+}(E)-I_{N}^{-}(E)
$$

It should be noted that the normalized spectral CD thus defined has a derivative line-shape similar to what is observed in the phenomenon of magnetic CD. $\underline{48}$ Using this definition, which corresponds to the anisotropy factor defined in Ref. 14, the ellipticity per unit absorbance can be determined at the exciton resonance as $\Theta=P(E) \ln 10 / 4\left(180^{\circ} / \pi\right) !{ }^{14}$ It is straightforward to show that in the limit that the level splitting between the $F_{z_{B}}= \pm 1$ transitions is small compared to the LW, the maximum spectral CD signal occurs at energy $\pm \sigma$ above and below the centroid $E_{c}$ of the two transitions.

Figure 5 shows spectral CD results calculated using the material parameters of Fig 4(C,D), which reflect a degenerate bright triplet finestructure at $\boldsymbol{K}=0$. Panel (A) shows CD spectra calculated for fixed azimuth and polar angles of 45 degrees as defined in the schematic in Fig. 4 (A), for several values of the $L W=10,20,30 \mathrm{meV}$. The spectra exhibit the expected derivitive line shape function centered about the $\boldsymbol{K}=0$ energies of the $F_{z_{B}}= \pm 1$ states. Panel (B) shows the maximum CD versus polar angle $\theta$ for fixed azimuth angle $\phi=45^{\circ}$. 

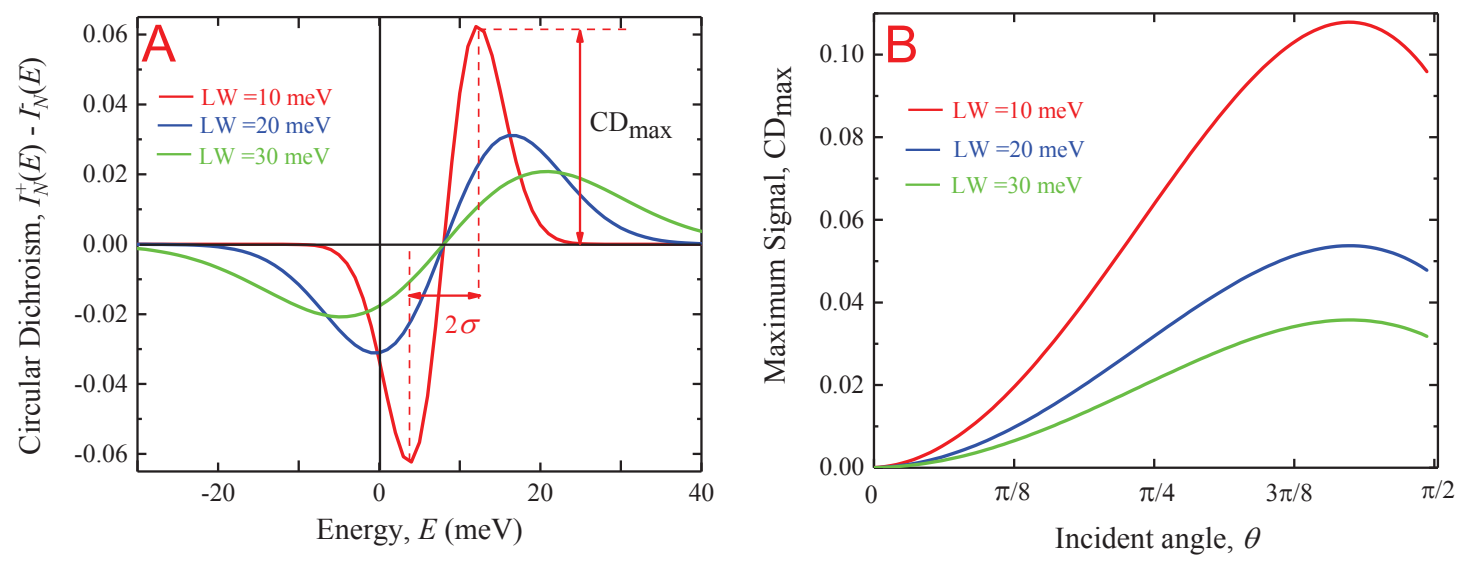

Figure 5. Spectral CD signal in 2D perovskite. Calculations represent the material parameters of Figure 4(C,D). Panel A shows the CD spectra $P(E)=I_{+}(E)-I_{-}(E)$ for fixed azimuth $\phi=45^{\circ}$ and polar angle $\theta=45^{\circ}$ as defined in the schematic in Fig. 4A. Spectra are calculated assuming a Gaussian line-shape for three values of the full width at half maximum $\mathrm{LW}(\mathrm{LW}=2 \sqrt{2 \ln 2} \sigma)$ and are normalized to give a peak absorption $I^{ \pm}$of unity as described in the text. Transition energy is plotted relative to the energy of the dark exciton at $\boldsymbol{K}=0$. The zero crossing occurs at the average energy of the $F_{z_{B}}= \pm 1$ states. The maximum $|\mathrm{CD}|$ occurs at $\pm \sigma$ from the zero crossing and is marked for the $\mathrm{LW}=10 \mathrm{meV}$ curve in the figure. The value of the maximum $\mathrm{CD}$ on the higher energy side is shown in panel $\mathrm{B}$ as a function of polar angle $\theta$ for fixed azimuth angle $\phi=45^{\circ}$.

\section{SUMMARY AND CONCLUSIONS}

We have shown that perovskite layers can exhibit CD which is observable in the off-normal optical excitation configuration and which does not require either an external magnetic field or chiral molecules on the layer surface. Polarization dependent absorption is expected to occur due to the combined effect of Rashba splitting, in-plane crystal symmetry breaking, and the effect of the exciton momentum on its fine structure. The fine structure can be understood in terms of Zeeman splitting due to a $\boldsymbol{K}$-dependent effective magnetic field, which can lead to splitting of helical excitonic states in systems of orthorhombic or lower crystal symmetry that exhibit Rashba spin splitting effects. The selective coupling of these helical exciton states to helical light is responsible for the c circular dichroism.

Using available measured values for the exchange and Rashba parameters determined 
experimentally in lead-iodide based 2D HOIS systems $35, \underline{36}, 46.47$, we showed that CD on the order of $10 \%$ for spectrally resolved fine structure transitions can occur for top illumination conditions in orthorhombic perovskites with inversion symmetry breaking normal to the 2D layers. For optimal side illumination conditions, spectrally resolved exciton fine structure levels may exhibit CD approaching 100\%.

Several effects have been shown to reduce the degree of observable CD. The principle limitation of the CD signal magnitude is the out-of-plane propagation of light given the assumption that the sample is illuminated from the top surface. Refractive steering of the light reduces the CD signal relative to optimized side illumination conditions by about an order of magnitude. Second, while the largest CD signal occurs for a pure 2D Dresselhauslike angular momentum texture, a mixed 2D Rashba and 2D Dresselhaus character reduces its magnitude. Additionally, the CD is shown to be reduced by crystal field splitting of the bright triplet exciton levels. due to the decrease in their mixing as the triplet degeneracy is already broken. Finally, in the case that the fine structure transitions are not individually spectrally resolved, the observable CD is reduced by line broadening effects.

Our calculations demonstrate that even with spectral line broadening effects at the level of tens of meV, CD should be readily observable in difference spectra which take the form of a line-shape derivative function, familiar from magnetic CD studies. Moreover, under optimized side illumination conditions, the CD is expected to be larger by an order of magnitude relative to the CD observable under top illumination conditions. Our results demonstrate the intriguing possibility of observing circular dichroism in non-chiral perovskite structures such layered-2D perovskites and nanoplatelets.

ACKNOWLEDGEMENTS P.C.S. and Z.V.V. acknowledge support from the Center for Hybrid Organic Inorganic Semiconductors for Energy (CHOISE) an Energy Frontier Research Center funded by the Office of Basic Energy Sciences, Office of Science within the US Department of Energy through contract number DE-AC36-08G028308. Al.L.E. acknowledges support from the US Office of Naval Research and the Laboratory-University Collaboration Initiative (LUCI) program of the DoD Basic Research Office. The authors acknowledge Dr. Haoliang Liu for useful discussions, Aditi Chandrashekar for assistance with manuscript preparation and Prof. D.J. Norris for a critical reading of the manuscript and valuable comments. 
The authors declare no competing financial interests.

Electronic Supplementary Material Available: Diagonalization of the exciton Hamiltonian and details of the CD calculation including analysis of polarization vectors.

[1] Sir William Thomson Lord Kelvin "The Molecular Tactics of a Crystal". Clarendon Press, Oxford 1894.

[2] N. Berova and K. Nakanishi, "Circular Dichroism: Principles and Applications", John Wiley \& Sons, New York, 2nd Ed., 2000.

[3] M. P. Moloney, Y. K. Gun'ko, J. M. Kelly, "Molecules on CdS", Chem. Commun. 2007, 7345, 3900-3902.

[4] A. Ben-Moshe, A. Teitelboim, D. Oron, and G. Markovich, "Probing the Interaction of Quantum Dots with Chiral Capping Molecules Using Circular Dichroism Spectroscopy", Nano Lett., 2016, 16, 7467-7473.

[5] C. Chen, L. Gao, W. Gao, C. Ge, X. Du, Z. Li, Y. Yang, G. Niu \&J. Tang, "Circularly polarized light detection using chiral hybrid perovskite" Nat. Commun., 2019 , 10:1927. https://doi.org/10.1038/s41467-019-09942-z.

[6] J. Ahn, S. Ma, J-Y. Kim, J. Kyhm, W. Yang, J. A. Lim, N. A. Kotov, and J. Moon, "Chiral 2D Organic Inorganic Hybrid Perovskite with Circular Dichroism Tunable Over Wide Wavelength Range" J. Am. Chem. Soc., 2020 , 142, 4206-4212.

[7] L. Wang, Y. Xue, M. Cui, Y. Huang, H. Xu, C. Qin, J. Yang, H. Dai and M. Yuan, "A Chiral Reduced-Dimension Perovskite for an Efficient Flexible Circularly Polarized Light Photodetector" Angew. Chem., 2020 , 132, 6504-6512.

[8] J. Han, S. Guo, H. Lu, S. Liu, Q. Zhao, and W. Huang, "Recent progress on circularly polarized luminescent materials for organic optoelectronic devices", Adv. Opt. Mater., 2018, 6, 1800538 .

[9] J. Ma, C. Fang, C. Chen, L. Jin, J. Wang, S. Wang, J. Tang, and D. Li, "Chiral 2D Perovskites with a High Degree of Circularly Polarized Photoluminescence" ACS Nano, 2019, 13, 36593665 .

[10] Y. Shi, P. Duan, S. Huo, Y. Li, and M. Liu, "Endowing Perovskite Nanocrystals with Circularly Polarized Luminescence" Adv. Mater., 2018, 30, 1705011. 
[11] H. Lu, J. Wang, C. Xiao, X. Pan, X. Chen, R. Brunecky, J. J. Berry, K. Zhu, M. C. Beard, Z. V, Vardeny, "Spin-dependent charge transport through 2D chiral hybrid lead-iodide perovskites" Sci. Adv., 2019, 5 : eaay0571.

[12] B. P. Bloom, V. Kiran, V. Varade, R. Naaman and D. H. Waldeck, "Spin Selective Charge Transport through Cysteine Capped CdSe Quantum Dots", Nano Lett., 2016, 16, 4583-4589.

[13] G. Long, C. Jiang, R. Sabatini, Z. Yang, M. Wei1, L. N. Quan, Q. Liang, A. Rasmita, M. Askerka, G. Walters, X. Gong, J. Xing, X. Wen, R. Quintero-Bermudez, H. Yuan, G. Xing, X. R. Wang, D. Song, O. Voznyy, M. Zhang, S. Hoogland, W. Gao, Q. Xiong and E. H. Sargent, "Spin control in reduced-dimensional chiral perovskites" Nat. Photonics, 2018, 12 528-533.

[14] G. Long, R. Sabatini, M. I. Saidaminov, G. Lakhwani, A. Rasmita, X. Liu, E. H. Sargent, and W. Gao, "Chiral-perovskite optoelectronics", Nat. Rev., 2020, 5, 423-439.

[15] W. Kaminsky, "Experimental and phenomenological aspects of circular birefringence and related properties in transparent crystals", Rep. Prog. Phys., 2000, 63, 1575-1640.

[16] B. Koopmans, P. V. Santos, and M. Cardona, "Optical Activity in Semiconductors: Stress and Confinement Effects", Phys. Stat. Sol. B, 1998, 205, 419-463.

[17] A. Ben Moshe, D. Szwarcman and G. Markovich, "Size Dependence of Chiroptical Activity in Colloidal Quantum Dots" ACS Nano, 2011, 5, 9034-9043.

[18] U. Tohgha, K. K. Deol, A. G. Porter, S. G. Bartko, J. K. Choi, B. M. Leonard, K. Varga, J. Kubelka, G. Muller and M. Balaz, "Ligand Induced Circular Dichroism and Circularly Polarized Luminescence in CdSe Quantum Dots" ACS Nano, 2013, 7, 11094-11102.

[19] Z. N. Georgieva, B. P. Bloom, S. Ghosh, and D. H. Waldeck, "Imprinting Chirality onto the Electronic States of Colloidal Perovskite Nanoplatelets" Adv. Mater., 2018, 30, 1800097.

[20] J. Ahn, E. Lee, J. Tan, W. Yang, B. Kim and J. Moon, "A new class of chiral semiconductors: chiral-organic-molecule-incorporating organicinorganic hybrid perovskites" Mater. Horiz., 2017, 4, 851-856.

[21] A. Ben-Moshe, A. O. Govorov and G. Markovich, "Enantioselective Synthesis of Intrinsically Chiral Mercury Sulfide Nanocrystals", Angew. Chem., Int. Ed., 2013, 52, 1275-1279.

[22] Y. Zhou, M. Yang, K. Sun, Z. Tang and N. A. Kotov, "Similar Topological Origin of Chiral Centers in Organic and Nanoscale Inorganic Structures: Effect of Stabilizer Chirality on Optical Isomerism and Growth of CdTe Nanocrystals" J. Am. Chem. Soc., 2010, 132, 60066013. 
[23] Y. Dong, Y. Zhang, X. Li, Y. Feng, H. Zhang, and J. Xu, "Chiral Perovskites: Promising Materials toward Next-Generation Optoelectronics" Small, 2019, 15, 1902237.

[24] D. G. Billing, A. Lemmerer, "Bis[(S)-b-phenethylammonium] tribromoplumbate(II)", Acta Crystallogr., Sect. E: Struct. Rep. Online, 2003, 59, m381-m383.

[25] D. G. Billing, A. Lemmerer, "Synthesis and crystal structures of inorganicorganic hybrids incorporating an aromatic amine with a chiral functional group" Cryst. Eng. Comm., 2006, 8, 686-695.

[26] M. V. Mukhina, V. G. Maslov, A. V. Baranov, A. V. Fedorov, A. O. Orlova, F. Purcell-Milton, J. Govan, and Y. K. Gun'ko, "Intrinsic Chirality of CdSe/ZnS Quantum Dots and Quantum Rods" Nano Lett., 2015, 15, 2844-2851.

[27] F. P. Milton, J. Govan, M. V. Mukhina and Y. K. Gun'ko, "A new class of chiral semiconductors: chiral-organic-molecule-incorporating organicinorganic hybrid perovskites" Nanoscale Horiz., 2016, 1, 14-26.

[28] J. Li, J. Li, R. Liu, Y. Tu, Y. Li, J. Cheng, T. He \& X. Zhu, "Autonomous discovery of optically active chiral inorganic perovskite nanocrystals through an intelligent cloud lab", Nat. Commun., 2020, 11:2046. doi.org/10.1038/s41467-020-15728-5.

[29] M. Isarov, L. Z. Tan, M. I. Bodnarchuk, M.V. Kovalenko, A. M. Rappe, and E. Lifshitz, "Rashba effect in a single colloidal CsPbBr3perovskite nanocrystal detected by magnetooptical measurements", Nano Lett., 2017,17, 5020-5026.

[30] J. F. Nye, "Physical properties of crystals: Their representations by tensors and matrices", Clarendon Press, 1957.

[31] M. V. Hobden, "Optical Activity in a Non-enantiomorphous Crystal Silver Gallium Sulphide" Nature, 1967, 216, 678.

[32] E. Plum, V. A. Fedotov, and N. I. Zheludev, "Optical activity in extrinsically chiral metamaterial" Appl. Phys. Lett. 2008, 93, 191911.

[33] E. Plum, V. A. Fedotov, and N. I. Zheludev, "Extrinsic electromagnetic chirality in metamaterials" JOSA A, 2009, 11, 074009.

[34] A. Y. Zhu, W. T. Chen, A. Zaidi, Y.-W. Huang, M. Khorasaninejad, V. Sanjeev, C.-W.Qiu, and F. Capasso, "Giant intrinsic chiro-optical activity in planar dielectric nanostructures" Light Sci. Appl., 2018, 7, 17158.

[35] Y. Zhai, S. Baniya, C. Zhang, J. Li, P. Haney, C.-X. Sheng, E. Ehrenfreund, Z. V. Vardeny, 
"Giant Rashba splitting in 2D organic-inorganic halide perovskites measured by transient spectroscopies"Sci. Adv., 2017, 3: e1700704.

[36] X. Liu, A. Chanana, U. Huynh, F. Xue, P. Haney, S. Blair, X. Jiang, X., and Z. V. Vardeny, "Circular photogalvanic spectroscopy of Rashba splitting in 2D hybrid organicinorganic perovskite multiple quantum wells", Nat. Commun., 2020, 11:323. https://doi.org/10.1038/s41467-019-14073-6

[37] Al. L. Efros, "Excitons in the structures with quantum wells" Sov. Phys. Semicond, 1986, 20, 808-812.

[38] X. L. Yang, S. H. Guo, F. T. Chen, K. W. Wong, and W. Y. Chin, "Analytical solution of a two-dimensional hydrogen atom. I. Nonrelativistic case", Phys. Rev. A, 1991, 43 1186-1196.

[39] N. C. Ritova, Vestnik of Moskow University, 1967, N3, 30. Translated to English in, https://arxiv.org/abs/1806.00976.

[40] L. V. Keldish, "Coulomb interactions in thin semiconductor and semimetal films", JETP Lett., 1979, 29, 716-719.

[41] R. Benchamekh, N. A. Gippius, J. Even, M.O. Nestoklon, J.-M. Jancu, S. Ithurria, B. Dubertret, Al. L. Efros, P. Voisin, "Tight-Binding Calculations of Image-Charge Effects in Colloidal Nanoscale Platelets of CdSe" Phys. Rev. B, 2014, 89 , 035307.

[42] M. A. Becker, R. Vaxenburg, G. Nedelcu, P.C. Sercel,A. Shabaev, M. J. Mehl, J.G. Michopoulos,S. G. Lambrakos, N. Bernstein, J.L. Lyons, T. Stöferle, R.F. Mahrt, M. V. Kovalenko, D.J. Norris, G. Raino, Al. L. Efros, "Bright triplet excitons in caesium lead halide perovskites." Nature, 2018, 553, 189-193.

[43] E.I. Rashba, and Al. L. Efros, "Orbital Mechanisms of Electron-Spin Manipulation by an Electric Field", Phys. Rev. Lett., 2003, 91, 126405.

[44] S. D. Ganichez and L. E. Golub, "Interplay of Rashba/Dresselhaus spin splittings probed by photogalvanic spectroscopy A review", Phys. Status Solidi B, 2014, 251, 1801-1823.

[45] P. C.Sercel, J. L. Lyons, N. Bernstein, Al. L. Efros, "Quasicubic model for metal halide perovskites", J. Chem. Phys., 2019, 151, 234106.

[46] K. Tanaka, T. Takahashi, T. Kondo, K. Umeda, K. Ema, T. Umebayashi, K. Asai, K. Uchida and N.Miura, "Electronic and Excitonic Structures of InorganicOrganic PerovskiteType Quantum-Well Crystal $\left(\mathrm{C}_{4} \mathrm{H}_{9} \mathrm{NH}_{3}\right)_{2} \mathrm{PbBr}_{4} "$ Jpn. J. Appl. Phys., Part 2, 2005, 44, 5923-5932. 
[47] T. Kataoka, T. Kondo, R. Ito, S. Kazuhito, K. Uchida, and N. Miura, "Magneto-optical study on excitonic spectra in $\left(\mathrm{C}_{6} \mathrm{H}_{1} 3 \mathrm{NH}_{3}\right)_{2} \mathrm{PbI}_{4} "$ Phys. Rev. B,1993, 47, 2010- 2018.

[48] M. Kuno, M. Nirmal,, M. G. Bawendi, Al. L. Efros, and M. Rosen, "Magnetic circular dichroism study of CdSe quantum dots", J. Chem. Phys., 1998, 108, 4242- 4247. 